\title{
EMG Speller with Adaptive Stimulus Rate and Dictionary Support
}

\author{
M. Vasiljevas, R. Turčinas, R. Damaševičius \\ Software Engineering Department, Kaunas University of Technology, Kaunas, Lithuania \\ Email: \{mindaugas.vasiljevas, rutenis.turcinas, robertas.damasevicius\}@ktu.lt
}

\begin{abstract}
Ambient Assisted Living (AAL) aims to improve the quality of daily life for all humans in different periods of life. Neural-Computer Interface (NCI) can be used within AAL environments to provide alternative communication means for impaired persons bypassing the need for speech and other motor activities. By monitoring, analyzing and responding to muscular activity (EMG signals) of users, NCI systems are able to monitor, diagnose and respond to the cognitive, emotional and physical states of users in real time. In this paper we analyze and develop a speller application based on the EMG interface. We analyze requirements for developing interfaces for disabled users and interfaces of known speller applications, and describe the development of the EMG-based speller as a benchmark application. The developed speller has adaptive stimulus rate and allows word selection from dictionary. We evaluate performance and usability of the developed speller using a set of empirical (accuracy, information transfer speed, input speed), ergonomic (NASA-TLX scale) and conceptual (humanistic intelligence) attributes.
\end{abstract}

\section{INTRODUCTION}

A mbient Assisted Living (AAL) environments comprise assisted technology devices, communication protocols and interfaces used to improve the quality of daily life for humans in different periods of their life [1]. Considering predictions of the demographic changes in society, AAL particularly focuses on elderly people though people with minor disabilities such as motor impairments can benefit, too. The AAL systems are user-centered and specifically are based on the concept of User Interfaces for All [2]. The concept aims at efficiently and effectively addressing the accessibility problems in human interaction with software applications and services while meeting the individual requirements of the users in general, including disabled and elderly people. Following the vision of e-Inclusion, the aim to "leave no-one behind" when enjoying the benefits of information and communication technology [3].

In the AAL environments, Neural-Computer Interfaces (NCI) can be used to provide alternative communication means for persons with disabilities bypassing the need for speech and other motor activities. NCI is similar to BrainComputer Interface (BCI) in methods used as well as in applications, however it uses the Electromyography (EMG) data rather than the Electroencephalography (EEG) data to establish an interface between human peripheral neural system and computers by recording electrical signals

This work was not supported by any organization governing muscular movements of a subject. The concepts are particularly suited to the needs of the handicapped as the cores of the smart environments and virtual reality applications. The state of a user is captured using sensors attached to the body. Then a physiological computing system creates a bio-cybernetic neurofeedback loop involving both human users and computers [4], which allows to produce a representation of the user's operational context. The loop may be designed to offer assistance if the user is frustrated or unable to perform the task due to excessive mental workload, adapt the level of challenge to sustain or increase task engagement if the user is bored or demotivated by the task, incorporating an emotional display element into the user interface, or alert for help if the user is not responsive [5].

When developing NCI systems for older adults (over 60) one has to consider that older people often have multiple, minor motor and cognitive function impairments or have slow control over their motor functions [6]. Given the often reported lower skin hydration in the elderly, the skin conductance is lower which leads to lower amplitudes and signal-to-noise ratio of measured EMG signals [7]. They also may also have slower control over muscle activities of the hands, fingers, etc. and decision-making may also be slower. For this group, the motivation to use NCI is completely different from the first group. Therefore, the design of a NCI for older adults should reflect their non-typical EMG profiles or slower response times.

Speller is a typical example of NCI/BCI application, which establishes a communication channel for people unable to use traditional keyboard and still remains a benchmark for BCI and NCI methods [8]. The speller is aimed to help those disabled persons unable to activate muscles traditionally used in communication (hands, tongue) to spell words by utilizing their neural activity. Typically, spellers use signal amplitude information, however integrating it with signal processing methods such as noise and dimensionality reduction methods and user intent prediction techniques can improve the results [9].

In this paper, we analyze the requirements for developing interfaces for impaired users and visual interfaces of known speller applications, describe the development of a speller as a typical benchmark application, and evaluate its performance and usability of the developed speller using a set of empirical (accuracy, information transfer speed, input speed), ergonomic (NASA-TLX scale) and conceptual (humanistic intelligence) attributes. 
The structure of the remaining parts of the paper is as follows. Section II analyzes the requirements for NCI systems and, specifically, NCI spellers. Section III discusses the interfaces of speller applications. Section IV describes the development of speller application. Section V presents the experimental results. Section VI evaluates the results. Finally, Section VII presents conclusions.

\section{ANALYSIS OF REQUIREMENTS FOR SPELLER APPLICATION}

The requirements for speller application can be categorized at different levels depending upon the physical abilities of its users [10]: 1) Users with no physical disability, who may use NCI for entertainment or other conditions where physical movement is restricted. 2) Users with minor impairments (such as older persons). 3) Users with severe physical disabilities, who may wish to use NCI as a secondary input. 4) Users who are almost locked-in (having limited muscle control), who may need to use NCI as a method for communication.

First, the speller must follow general requirements for smart systems to be integrated into the AAL environments. Next, the specific requirements for impaired users (and, specifically, for older persons) must be followed. Impaired users need assistance such as automatic learning of user's behavior to estimate his/her current needs.

Since humans often make mistakes or errors in interacting with machines, for any human-operated system, user interfaces should be designed such that prevent errors whenever possible; deactivate invalid commands; make errors easy to detect and show users what they have done; and allow undoes, reverse, correct errors easily [11].

For smart systems, the following principles (also called “operational modes") of Humanistic Intelligence Framework [12] must be satisfied:

1) Constancy: the interface should operate continuously to read signals from human to computer and to provide a constant user-interface.

2) Augmentation: the primary task is increasing the intelligence of the system rather than computing tasks.

3) Mediation: the interface mediates between human senses, emotions and perceptions and acts as information filter by blocking or attenuating undesired input to decrease negative effects of interaction (such as fatigue, information overload, etc.) as well as to increase positive effects (such as user satisfaction) by amplifying or enhancing desired inputs.

According to Lopes [13], the user interface for persons with disabilities must: support user variability allowing to provide the means to adapt to user-specific requirements; support of a wide range of input devices and output modes; provide minimal user interface design; promote interaction and retain user attention on the tasks; and provide strong feedback mechanisms that may provide rewarding schemes for correct behavior (results).

The requirements for interfaces for impaired users can be formulated as follows [1]: 1) Limited access to details: complex and vital details of the system have to be hidden to avoid user overwhelming and trapping. 2) Self-learning: detected common patterns in the behavior of the user should be used to automatically create rules or shortcuts that speed and ease up the use of the system. 3) System interruption: Impaired users have in most cases no idea how the system is working, therefore easy cancellation of system's activities must be ensured.

In the questionnaire-based study of potential BCI user requirements towards assisted technologies [14], the participants rated participants rated "functionality" (aka effectiveness) as the most important requirement, followed by "possibility of independent use" and "easiness of use".

\section{REVIEW OF SPELLER INTERFACES}

Many different variants of interfaces have been proposed and designed for speller, a de-facto benchmark application of $\mathrm{BCI} / \mathrm{NCI}$. Based on the complexity and visual representation of symbols to input, they can be categorized into the following classes:

Linear (or single character) speller: all symbols are shown and each symbol is flashed individually until symbol selection is done [15].

Matrix (or Row-Column) Speller: All letters are arranged in a matrix . First, speller flashes an entire column (Fig. 1, left) or row of characters (Fig. 1, right). Then, single letters are flashed in a sequence, and can be selected. Different matrix sizes can be used, e.g., a $6 \times 6$ matrix containing all 26 letters of the alphabet and 10 digits (0-9) [16], or even a full QWERTY keyboard [17].
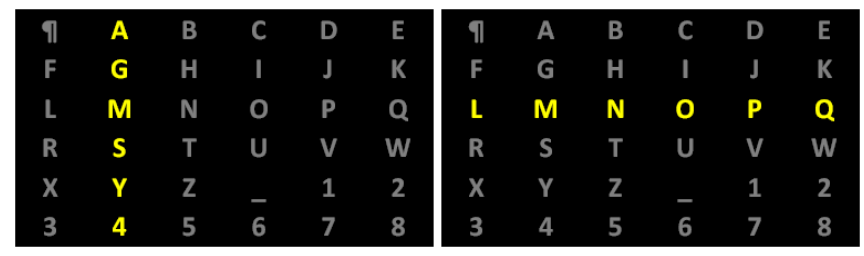

Fig. 1. Example of matrix speller interface.

Chekerboard Speller [18]: the $8 \times 9$ matrix is virtually superimposed on a checkerboard (Fig. 2, left), which the participants never actually see. The items in white cells of the $8 \times 9$ matrix are segregated into a white $6 \times 6$ matrix and the items in the black cells are segregated into a black $6 \times 6$ matrix. Before each sequence of flashes, the items in Fig. 2 (left) randomly populate the white or black matrix, respectively, as shown in Fig. 2 (middle). The checkerboard layout controls for adjacency-distraction errors, because adjacent items cannot be included in the same flash group. The users see random groups of six items flashing (as opposed to rows and columns) because the virtual rows and columns depicted in Fig. 2 (middle) flash. 


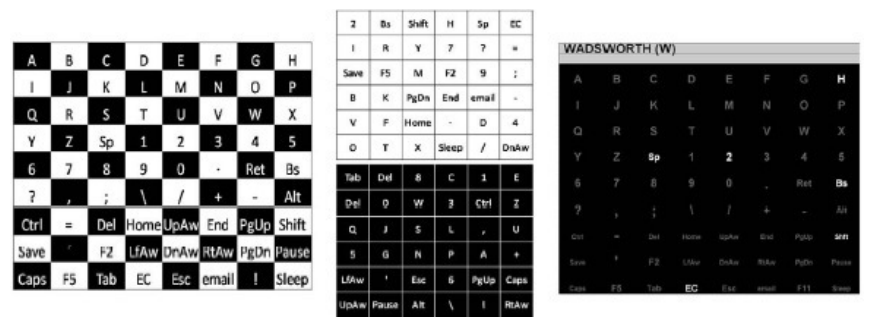

Fig. 2. Checkerboard intherface of the speller [18]

Frequency-based layout accounts for the relative frequency of character occurrence in a language as in Bremen SSVEP-based BCI-speller [19]. It has in the middle of the screen a virtual keyboard with 32 symbols (see Fig. 3) surrounded by five boxes flickering at different frequencies. These boxes correspond to commands for navigating the cursor (indicated by red color), and for selecting the intended character. The application starts with a cursor in the central position corresponding to the most frequent character in English (i.e., "E"). Letters with the higher frequency of occurrence are positioned closer to the center while the less frequent ones are further away. The user can navigate the cursor to the desired letter and confirm his/her choice with the "Select" command. The further the character is located from the center, the more command selections (cursor movements) are required.

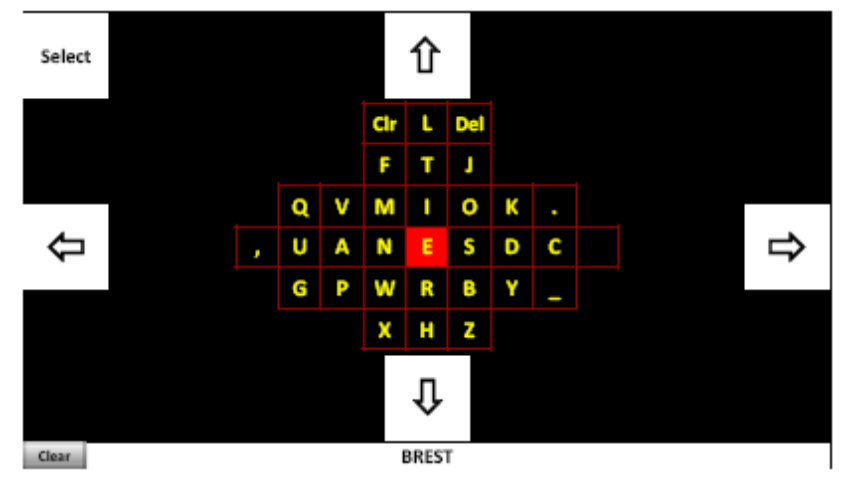

Fig. 3. Interface of the Bremen BCI speller [19].

Region-based Speller: groups of characters arranged into different regions, which contain different subsets of characters (Fig. 4). When the user confirms the selection of the group, the characters of the group are divided into new groups until the desired character is selected. Examples of such interface are 27 symbol triads [20] or 64 symbol quadrants [21].

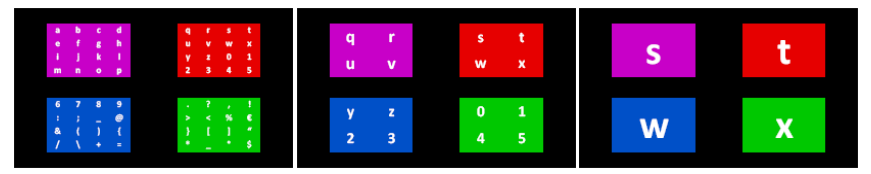

Fig. 4. Consecutive stages to select symbol in the quadrant region-based speller [21]
The Rotate-Extend (REx) paradigm [10] consists of a wheel divided into segments (see Fig. 5). An arrow in the centre of the wheel controls the selection of target segments. One mental class is used to control the rotation of the arrow, and the other class extends the arrow to select the target segment. Example of Rex interface is Hex-o-Spell speller [22], which allows 30 different characters to be typed in. The characters are shown in six adjacent hexagons distributed around a circle. Each hexagon contains five characters and a „go back" command. For the selection of the hexagons, there is an arrow in the center of the circle. After selection, the characters in all hexagons, except for the selected one disappear, while the remaining characters and the ,go back“ command are mapped into six hexagons around the circle. Using the same arrow-based strategy, the user selects the desired character or decides to go back to the previous level of the interface to correct a mistake. Another implementation of REx interface is Oct-o-spell, where a larger set of symbols is used [23].

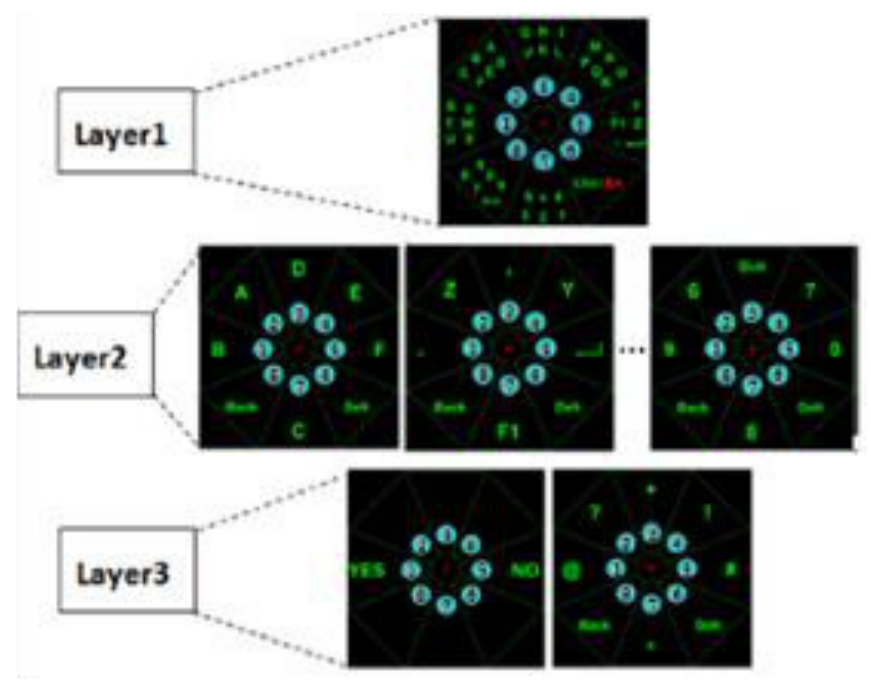

Fig. 5. Interface of Oct-o-spell speller [23]

The overview of visual interfaces of spellers can be summarized as follows. The interfaces can classified according to interface paradigm (linear/single, matrix, chekerboard, frequency layout, region-based, RotateExtend), stimulus type (the way each individual character changes, e.g., flashing frequency, color change, distance to target, etc.), stimulus rate (the speed at which individual characters change), stimulus pattern (grouping of symbols in interface), character set (usually includes all letters of the alphabet as well as additional symbols such as numbers, separation marks, etc., enhancing intelligence (additional techniques for improving accuracy of the system and rate of communication such as using language model, word autocomplete, spelling correction or word prediction). 


\section{DEVELOPMENT OF EMG SPELLER}

A NCI system generally comprises the following components: (i) a device that records the muscular activity signals; (ii) a signal preprocessor that reduces noise and artifacts; (iii) a decoder that classifies the de-noised signal into a control commands for (iv) an external device or application (e.g., a robotic actuator, a computer program etc.), which provides feedback to the user [24].

Our speller application has three layers as follows: 1) on the lowest layer, the physiological signal is sampled into a data stream of physiological data. Downsampling can be used to decrease amount of data and increase information processing speed at higher levels. 2) On the intermediate layer, data is aggregated and events corresponding to specific patterns of data are generated. Machine learning techniques such as artificial neural networks may be used to recognize such events and generate decisions. 3) On the highest layer, decisions are processed and used to generate control commands for external applications (systems).

The architecture of the developed speller application is shown in Fig. 6. The speller has 6 components: MainReader is responsible for control of data reader which is selected to use. ReaderAPI is public external interface of third-party EMG data reader modules. MainController is responsible for selected control module (executes commands). NiaReader is a third-party module implemented for the OCZ NIA data reader device. SpellingSquare is a third-party module implemented for text input in symbol matrix using the EMG-based commands.

The dashed rectangle separates system components from external components. Components inside the rectangle are considered as system components. Components outside the rectangle are considered as external components. System was developed with respect to maintenance so that external components were easy to add or remove. The external components are sensors (EMG readers), actuators (robot, etc.) controllers or external software. The NetBeans framework was used for development. It provides the opportunity to add third-party components on-demand.

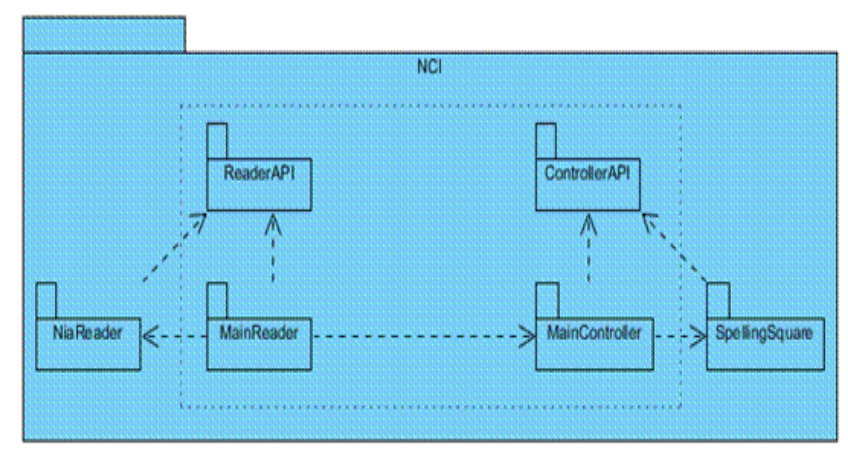

Fig. 6 Architecture of physiological computing system
The feedback to the user is an important aspect of the NCI as it provides the user with information about his/her mistakes as well as motivates the user to increase attention and engagement in the task. The main element of the developed speller application that supports feedback is visual interface (Fig. 7). It contains the representation of the symbol matrix (the size and character set of the matrix is adaptable). The red-colored column indicates the current position of the speller cursor. The cursor moves sequentially from column to column until the user activates the "Select" command. Next, the cursor moves through each symbol in the selected column. After another "Select" command the particular symbol is selected and appears in the text output area. The stimuli rate (the speed of the cursor can vary from 500 to $1500 \mathrm{~ms}$ ) depends upon the number of input mistakes the user does (the speed increases or decreases automatically to keep the number of mistakes low). The mistake is considered as the "Cancel" command, which exits the selected column or deletes the selected symbol.

The control commands are initiated by the movements of facial muscles (left eye blink for "select" and right eye blink for "cancel"). The user can see the EMG signal feedback in the EMG signal view area of the interface (Fig. 8). The particular control command is performed when the amplitude of the EMG signal is equal or higher than the specified threshold value (marked with yellow horizontal lines). The upper threshold (high positive amplitude) indicates the "Select" command, while the lower threshold (high negative amplitude) indicates the "Cancel" command. The threshold values can be adjusted by the user via settings.

The signal view of EMG, while spelling the word "hello", is presented in Figs. 8 \& 9. In Fig. 8, the word "hello" is spelled without mistakes. In Fig. 9, the same example is presented but in this case it contains a few mistakes (wrong selections). For correction of those mistakes cancellation commands must be performed. The spikes indicate the "select" command. One trial (selection of one character) contains two positive signal spikes, the first spike is for column selection, the second for letter selection in the corresponding column.

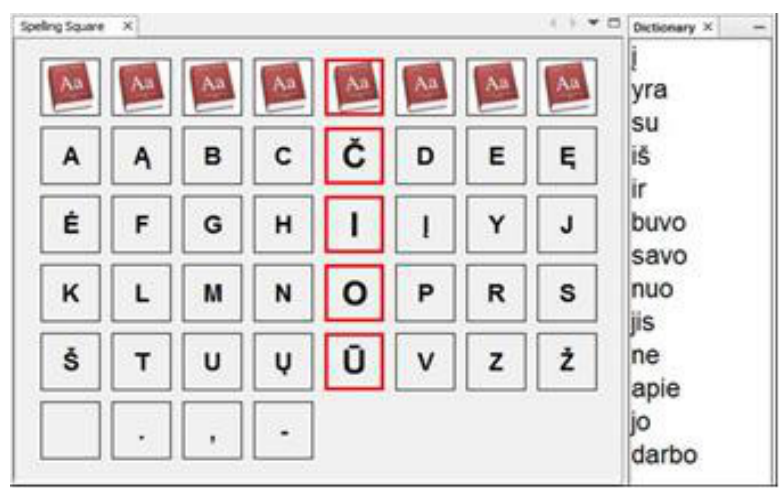

Fig. 7. Interface of developed speller application 


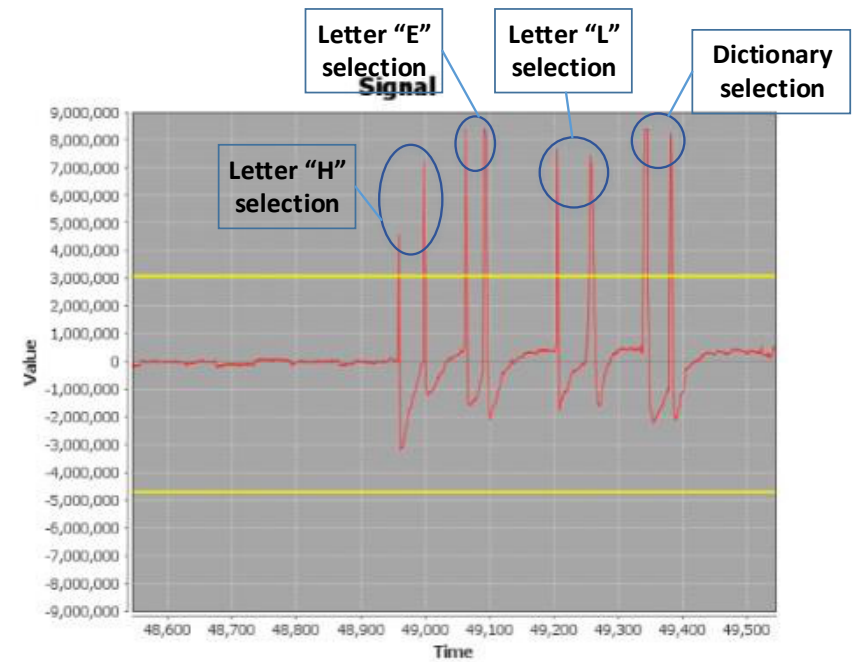

Fig. 8. Signal view of spelling word "hello". In this example no spelling mistakes were made and only three characters ("hel") were selected from the symbol matrix. Dictionary selection was made to complete the word.

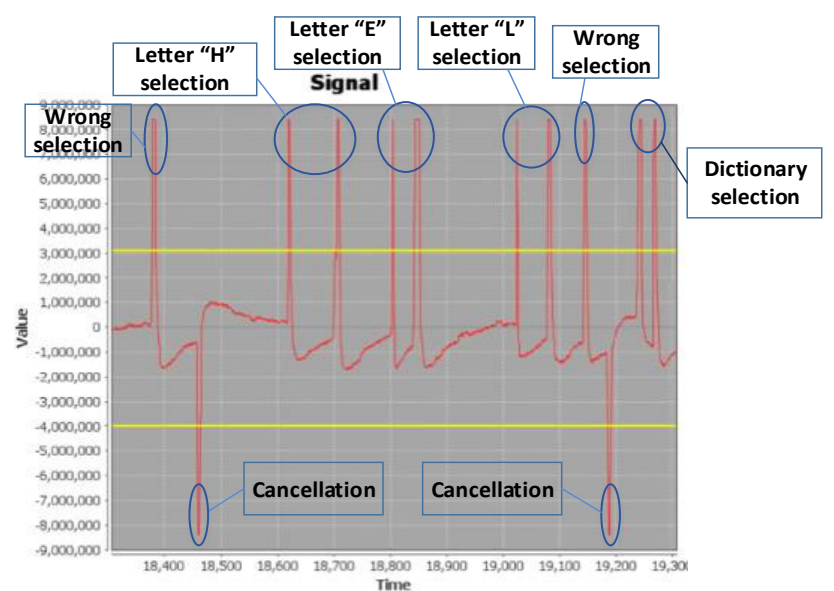

Fig. 9. Signal view of spelling word "hello". This example contains two spelling mistakes therefore after each wrong selection cancellation command was performed. Three characters ("hel") were selected from symbol matrix. Dictionary selection was made to complete the word.

The external dictionary can be used while entering symbols. The dictionary words are filtered using the already entered part of the word and shown to the user based on their frequency in the text corpora of the given language. The frequency value of the word is increased based on its usage frequency by the user. If the user enters a word absent in the dictionary, the dictionary is updated.

\section{V.EXPERIMENTAL SETTING}

The experiments we performed with 5 subjects ( 3 males, 2 females), aged 24-54 (mean = 33) year. Subjects did not have any neurological abnormalities, reported normal or corrected to normal vision, and did not use medication. All subjects gave informed consent prior to the experiment.

EMG was recorded using OCZ Neuro Impulse Actuator equipment, which employs three electrodes across the forehead (see Fig. 10). It uses carbon interface fibers injected into a soft plastic as sensors to capture a combination of muscle, skin, and nerve biopotentials.

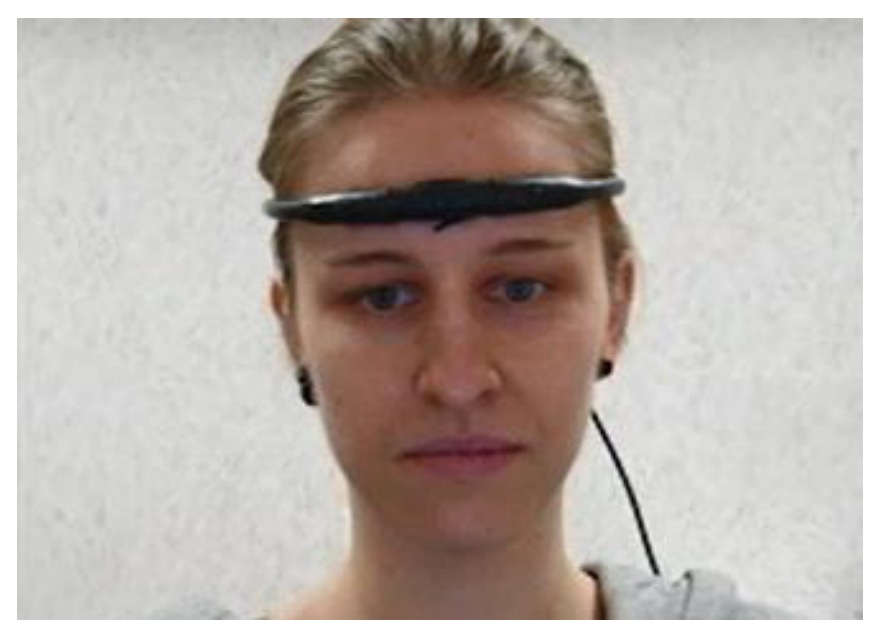

Fig. 10. The test subject with OCZ NIA device

The test subjects were seated in front of a table, $100 \mathrm{~cm}$ away from the liquid crystal display (LCD) showing stimuli. Visual stimuli were presented on a 13.3" size LCD screen with $1360 \times 768$ pixel resolution and a refresh rate of $60 \mathrm{~Hz}$. Contrast and brightness are set to maximum. The size of each character was $1.5 \times 1.5 \mathrm{~cm}\left(0.86 \times 0.86^{\circ}\right.$ visual angle $)$ and the entire speller matrix was $9.5 \times 13 \mathrm{~cm}\left(5.44 \times 7.42^{\circ}\right.$ visual angle). Stimuli consisted of intensifications of the rows and columns in sequential order. Intensification was achieved by increasing the size of all characters in the row or column with a factor 500 for $1500 \mathrm{~ms}$. A trial is defined here as spelling of one character. All trials started with the speller being displayed on the screen, together with an instruction indicating which letter to select. Each stimulation sequence was followed by feedback on the screen, showing which letter or group of letters had been selected.

Three text paragraphs were given to the experiment participants. Their task was to input the proposed text paragraphs using speller. All text paragraphs were presented in Lithuanian. The first text paragraph contained 126 characters and its content covered a daily conversation. The second text paragraph contained 111 characters and its content covered a scientific speech. The third text paragraph contained 120 characters and covered a scientific speech with mathematical equations. Each experiment participant repeated the experiment 4 times with different speller settings. The first test was made with basic speller settings. The second test was made with adaptable stimulus time (the stimulus time varied from $500 \mathrm{~ms}$ to $1500 \mathrm{~ms}$ depending on the amount of mistakes). The third test was made with dictionary. The fourth test was made with both dictionary and adaptable stimulus time. The average accuracy, input speed and bit rate values were calculated. The results of experiments are presented in Section VI. 


\section{Evaluation of RESUlts}

Quantitatively, the performance of speller application can be evaluated using accuracy, information transfer speed and input speed metrics. Accuracy is calculated as the percentage of correct decisions. Information transfer rate (or bit rate) indicates how much information can be communicated per time unit and is calculated using Walpaw's formula [25]. Finally, input speed is measured as the average time required to input a set of benchmark texts.

The accuracy results of BCI/NCI-based speller applications achieved by other authors are within $80-95 \%$ range (80\% using EEG-based P300 speller [26], 82.77\% using ECoG [9], 84.22\% using invasive BCI [27], 87.58\% using SSVEP based BCI [17], 87.8\% for EOG-based speller [28], 89.5\% [29], 91.80\% [30], 94.8\% for RSVP based speller [31], 90.81\% for SSVEP-based speller [32], 95.18\% for Oct-o-spell [23]).

The information transfer rate (aka bit rate) of the BCI/NCI-based speller applications achieved by other authors are within 7-41 bits/min (7.43 bits/min [33], 17.13 bits/min [29], $19.18 \mathrm{bits} / \mathrm{min}$ [30], 11.58-37.57 bits/min [32], 40.72 using SSVEP based BCI [17], 41.02 using ECoG [9]).

The symbol input speed of the BCI/NCI-based speller applications achieved by other authors are within 1-12 CPM (1.38 CPM for EOG-based speller [28], 1.43 CPM for RSVP based speller [31], 4.33 CPM [30], 4.91 CPM [32], 9.39 CPM using SSVEP based BCI [17], 10.16 CPM [23], 12.75 CPM [34]).

The results of the evaluation developed speller are given in Table I and summarized in Figs. 11-13. Best results in terms of both average and peak information transfer rate and input rate values are achieved when adaptable stimulus rate is used together with the dictionary. However, higher input speed inevitably lead to larger number of errors, therefore, accuracy is lower than using the speller with basic settings.

TABLE I.

EVALUATION OF SPELLER APPLICATION

\begin{tabular}{|c|c|c|}
\hline Quantitative metric & Average Value & Peak value \\
\hline \multicolumn{3}{|c|}{ BASIC SETTINGS } \\
\hline Accuracy & 96.29 & 98.25 \\
\hline Information transfer rate & 34.78 & 41.83 \\
\hline Input speed & 6.37 & 7.57 \\
\hline \multicolumn{3}{|c|}{ ADAPTABLE STIMULUS RATE } \\
\hline Accuracy & 88.61 & 93.64 \\
\hline Information transfer rate & 42.53 & 49.79 \\
\hline Input speed & 8.19 & 9.60 \\
\hline \multicolumn{3}{|c|}{ WITH DICTIONARY } \\
\hline Accuracy & 92.65 & 96.06 \\
\hline Information transfer rate & 43.55 & 49.26 \\
\hline Input speed & 8.22 & 9.35 \\
\hline \multicolumn{3}{|c|}{ WITH ADAPTABLE STIMULUS RATE AND DICTIONARY } \\
\hline Accuracy & 89.16 & 92.53 \\
\hline Information transfer rate & 58.69 & 65.53 \\
\hline Input speed & 11.35 & 12.42 \\
\hline
\end{tabular}

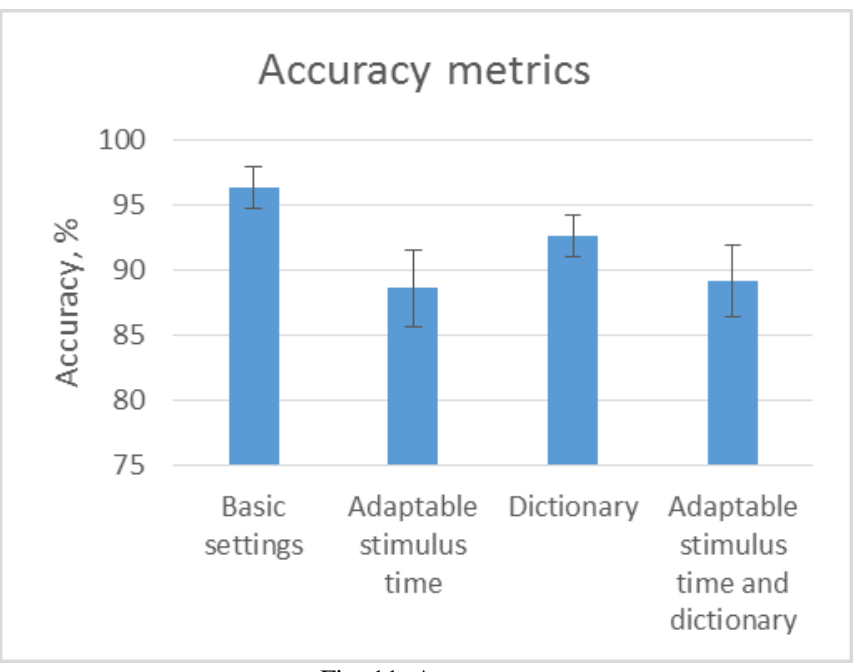

Fig. 11. Accuracy.

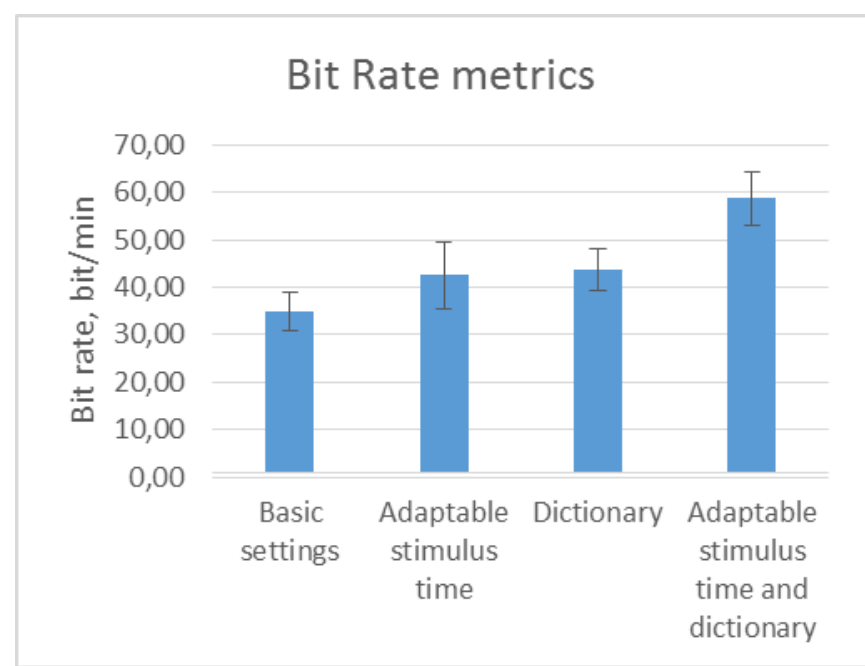

Fig. 12. Information transfer rate

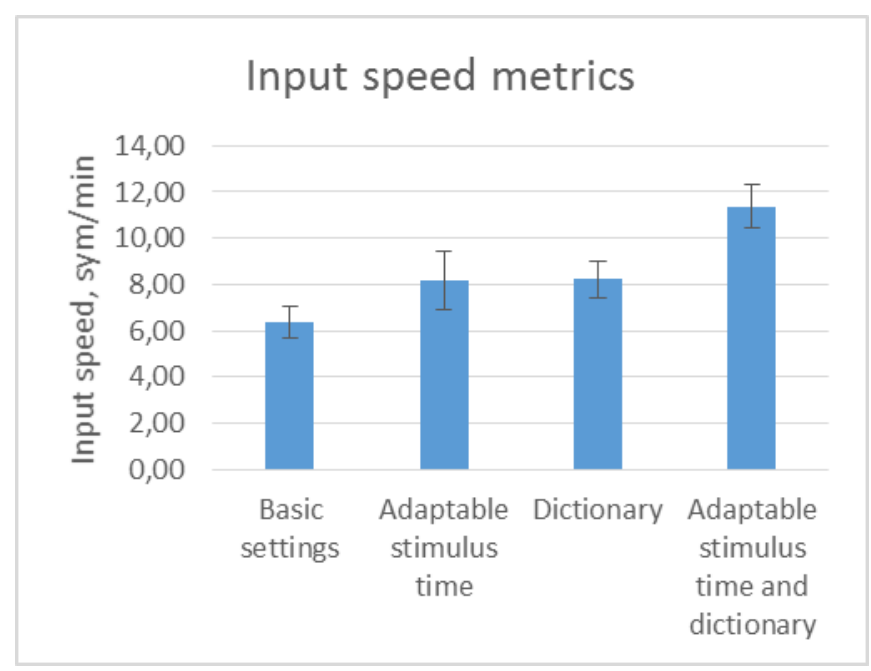

Fig. 13. Input speed

Qualitatively, the speller application can be evaluated based on user's mental workload required to work with this application. Here we use the NASA Task Load Index (TLX) questionnaires [35], a multi-dimensional rating procedure with six subscales: Mental Demands, Physical Demands, Temporal Demands, Performance, Effort, and Frustration. 
TABLE II.

EVALUATION OF SPELLER APPLICATION USING NASA-TLX INDEX

\begin{tabular}{|l|c|}
\hline NASA-TLX scale & Average value \\
\hline Mental Demands & 52 \\
\hline Physical Demands & 66.6 \\
\hline Temporal Demands & 45 \\
\hline Performance & 48 \\
\hline Effort & 67 \\
\hline Frustration & 55 \\
\hline
\end{tabular}

The users' subjective workload was assessed with the NASA Task Load Index (TLX), which identifies (1) the overall workload in the different tasks and (2) the main sources of workload. Workload in the TLX is defined as a "hypothetical construct that represents the cost incurred by a human operator to achieve a particular level of performance." The TLX is specifically adequate when interested in detecting the sources of workload. Workload is estimated with six subscales (mental, physical, and temporal demand and performance, effort, and frustration). Participants rated subjective workload for each dimension on twenty step bipolar scales with scores from 0 to 100 . A weighting procedure was used to combine the six individual ratings into a global score. To do so, the six scales were combined to 14 pairs and subjects had to indicate which scale of the pair contributed more to their workload. A weighted average technique was then used to compute an overall measure of workload (between 0 and 100) and the relative contribution of each subscale to overall workload.

The NASA-TLX contains six factors (shown in Table III), each of which has 20 levels and is scored from 0 to 100 . Small score represents low workload and vice versa. The speller application was evaluated by 5 healthy subjects and the results are presented in Table III. Main sources of workload were physical demands and effort. The average score for the workload factors are less than 56\%. This observation indicates that the speller interface is acceptable for all subjects. High scores for "Temporal demand" given by most of the subjects indicate that the speed of the system must be improved further. Low scores for "Frustration" indicate that subjects are interested in using speller application and that the results meet their expectations.

TABLE IV.

EVALUATION OF SPELLER APPLICATION BASED ON THE MANN'S ATTRIBUTES OF HUMANISTIC-INTELLIGENCE SYSTEM

\begin{tabular}{|c|c|c|}
\hline Attribute & Evaluation & Comment \\
\hline Unmonopolizing & Yes & $\begin{array}{l}\text { Speller does not cut the user off } \\
\text { from the outside world }\end{array}$ \\
\hline Unrestrictive & Yes & $\begin{array}{l}\text { User can use other channels of } \\
\text { communication at the same time } \\
\text { while using the speller }\end{array}$ \\
\hline Observable & Yes & $\begin{array}{l}\text { Speller can get the user's attention } \\
\text { continuously if and the output } \\
\text { medium is constantly perceptible }\end{array}$ \\
\hline Controllable & Yes & User can control the speller anytime \\
\hline Attentive & Yes & $\begin{array}{l}\text { Speller is context aware, } \\
\text { multimodal, and multisensory }\end{array}$ \\
\hline Communicative & Yes & $\begin{array}{l}\text { Speller allows to communicate } \\
\text { directly to other users or spellers }\end{array}$ \\
\hline
\end{tabular}

Conceptually, the speller application can be evaluated based on the attributes (Unmonopolising, Unrestrictive, Observable, Controllable, Attentive, Communicative), which every humanistic-intelligence system must have, as formulated by Mann [12] (see Table IV).

\section{CONCLUSION}

In this paper we have described the development of the speller application for an assisted living environment using the EMG interface. This system is controlled by voluntary muscular movements, particularly the orbicular ones (i.e., eye blinking). These movements are translated into instructions which allow the text input.

The developed speller application is adaptive (text input speed can be adapted dynamically in response to the user's state) and intelligent (machine learning techniques are used to analyze input data to achieve high accuracy of selection as well as to increase text input speed by using word complete and word frequency features).

The speller has been evaluated empirically (using accuracy, information transfer speed and input speed), ergonomically (using the NASA-TLX scale of subjective workload) and conceptually (using the attributes of Mann's Humanistic Intelligence Framework [12]).

The achieved empirical results are within range of results achieved by other authors, while the ergonomic evaluation suggests that users are interested in using speller application and that the results meet their expectations, yet the speed of the system as well as the usability of its interface could be improved further.

This system can aid people with reduced mobility, extending the time that older people and disabled people can live in their home environment, increasing their autonomy and their confidence.

Future work will focus on going beyond low-level typing to graphical-symbol matrix that allows selection of concepts rather than stand-alone letters. Also the performance characteristics of the speller application will be researched further aiming to maximize usability of the product both in terms of increased speed as well as better ease of use.

\section{ACKNOWLEDGMENT}

The work described in this paper has been carried out within the framework the Operational Programme for the Development of Human Resources 2007-2013 of Lithuania „Strengthening of capacities of researchers and scientists“ project VP1-3.1-ŠMM-08-K-01-018 „Research and development of Internet technologies and their infrastructure for smart environments of things and services" (2012-2015), funded by European Social Fund (ESF).

The authors would like to acknowledge the contribution of the COST Action IC1303 - Architectures, Algorithms and Platforms for Enhanced Living Environments (AAPELE). 


\section{REFERENCES}

[1] A. Marinc, C. Stocklöw, A. Braun, C. Limberger, C. Hofmann, and A. Kuijper, „Interactive personalization of ambient assisted living environments," Proc. of the 2011 Int. Conf. on Human interface and the management of information - Volume Part I (HI'11), LNCS vol. 6771, Springer-Verlag, Berlin, Heidelberg, 2011, 567-576. DOI: 10.1007/978-3-642-21793-7_64

[2] C. Stephanidis, "Towards User Interfaces for All: Some Critical Issues," Advances in Human Factors/Ergonomics, 01/1995, 20:137142. DOI:10.1016/S0921-2647(06)80024-9

[3] C. Zickler, A. Riccio, F. Leotta, S. Hillian-Tress, S. Halder, E. Holz, P. Staiger-Sälzer, E.J. Hoogerwerf, L. Desideri, D. Mattia, and A. Kübler, "A brain-computer interface as input channel for a standard assistive technology software," Clinical EEG and Neuroscience, 2011, 42(4), 236-44. DOI:10.1177/155005941104200409

[4] N.B. Serbedzija, and S.H. Fairclough, "Biocybernetic loop: From awareness to evolution," IEEE Congress on Evolutionary Computation, 2009, 2063-2069. DOI: 10.1109/CEC.2009.4983195

[5] S.H. Fairclough, "Fundamentals of physiological computing", Interacting with Computers (IWC), 2009, 21(1-2):133-145.

[6] R. Adams, R. Comley, and M. Ghoreyshi, "The Potential of the BCI for Accessible and Smart e-Learning," Proc. of the 5th Int. Conference on Universal Access in Human-Computer Interaction. Part II: Intelligent and Ubiquitous Interaction Environments (UAHCI '09), 2009, 467-476. DOI: 10.1007/978-3-642-02710-9_51

[7] J. Kemp, O. Després, T. Pebayle, and A. Dufour, "Age-related decrease in sensitivity to electrical stimulation is unrelated to skin conductance: an evoked potentials study," Clinical Neurophysiology, 2014, 125(3):602-7. DOI: 10.1016/j.clinph.2013.08.020

[8] H. Cecotti, "Spelling with non-invasive Brain-Computer Interfaces Current and future trends", Journal of Physiology-Paris, 2011, 105(1-3), 106-114. DOI: 10.1016/j.jphysparis.2011.08.003

[9] W. Speier, I. Fried, and N. Pouratian, "Improved P300 speller performance using electrocorticography, spectral features, and natural language processing," Clinical Neurophysiology, 2013, 124(7), 13211328. DOI: 10.1016/j.clinph.2013.02.002

[10] M. Quek, J. Höhne, R. Murray-Smith, and M. Tangermann, "Designing Future BCIs: Beyond the Bit Rate", in Allison, B., Dunne, S., Leeb, R., Millan, J.D.R. and Nijholt, A. (eds.), Towards Practical Brain-Computer Interfaces: Bridging the Gap from Research to Real-world Applications, Springer, 2013, 173-196. DOI: 10.1007/978-3-642-29746-5_9

[11] J. Johnson, Designing with the Mind in Mind: a Simple Guide to Understanding User Interface Design Rules. Morgan Kaufmann, Burlington, 2011.

[12] S. Mann, "Wearable computing: Toward humanistic intelligence", IEEE Intelligent Systems, 2001, 16(3): 10-15. DOI: $10.1109 / 5254.940020$

[13] J.B. Lopes, "Designing user interfaces for severely handicapped persons," Proc. of the 2001 EC/NSF workshop on Universal accessibility of ubiquitous computing: providing for the elderly (WUAUC'01), ACM, New York, NY, USA, 2001, 100-106. DOI: $10.1145 / 564526.564553$

[14] C. Zickler, V. Kaiser, A. Al-Khodairy, S. Kleih, A. Kübler, M Malavasi, D. Mattia, S. Mongardi, C. Neuper, M. Rohm, R. Rupp, P. Staiger-Sälzer, and E.-J. Hoogerwerf, "BCI-Applications: Requirements of Disabled End-Users and Professional Users", First TOBI Workshop, Graz, Austria, February 2010.

[15] R. Ortner, R. Prueckl, V. Putz, J. Scharinger, M. Bruckner, A. Schnuerer, and C. Guger, "Accuracy of a P300 Speller for Different Conditions: A Comparison," Proc. of the 5th Int. Brain-Computer Interface Conference, 2011, Graz, Austria, p. 196.

[16] L. A. Farwell and E. Donchin, "Talking off the top of your head: toward a mental prosthesis utilizing event-related brain potentials," Electroencephalography and clinical neurophysiology, 70(6), 510523, Dec 1988. DOI: 10.1016/0013-4694(88)90149-6

[17] H.-J. Hwang, J.-H. Lim, Y.-J. Jung, H. Choi, S.W. Lee, and C.-H. Im, "Development of an SSVEP-based BCI spelling system adopting a QWERTY-style LED keyboard," Journal of Neuroscience Methods, 208(1), 2012, 59-65. DOI: 10.1016/j.jneumeth.2012.04.011
[18] G. Townsend, B. K. LaPallo, C. B. Boulay, D. J. Krusienski, G. E. Frye, C. K. Hauser, N. E. Schwartz, T. M. Vaughan, J. R. Wolpaw, and E. W. Sellers, "A novel p300-based brain-computer interface stimulus presentation paradigm: moving beyond rows and columns," Clinical Neurophysiology, 121(7), 2010, 1109-1120. DOI: 10.1016/j.clinph.2010.01.030

[19] I. Volosyak, H. Cecotti, D. Valbuena, and A. Gräser, "Evaluation of the Bremen SSVEP Based BCI in Real World Conditions", in Proc. of the 11th Int. Conference on Rehabilitation Robotics, Kyoto, Japan, 2009, 322-331. DOI: 10.1155/2012/967305

[20] T. D’Albis, R. Blatt, R. Tedesco, L. Sbattella, and M. Matteucci, "A predictive speller controlled by a brain-computer interface based on motor imagery," ACM Transaction of Computer-Human Interaction, 19(3):20:1-20:25, October 2012. DOI: 10.1145/2362364.2362368

[21] H. Segers, A. Combaz, N.V. Manyakov, N. Chumerin, K. Vanderperren, S. Van Huffel, and M.M. Van Hulle, "Steady State Visual Evoked Potential (SSVEP)-Based Brain Spelling System with Synchronous and Asynchronous Typing Modes," 15th Nordic-Baltic Conference on Biomedical Engineering and Medical Physics (NBC 2011), Aalborg, Denmark, 14-17 June 2011, 164-167. DOI: 10.1371/journal.pone.0073691

[22] M. Treder, and B. Blankertz. "(C)overt attention and visual speller design in an ERP-based brain-computer interface," Behavioral and Brain Functions, 2010, 6, 1-13. DOI: 10.1186/1744-9081-6-28

[23] C. Cheng, J. Yang, Y. Huang, J. Li, and B. Xia, "A Cursor Control Based Chinese-English BCI Speller," Neural Information Processing, Lecture Notes in Computer Science, 8226, 2013, 403-410. DOI: 10.1007/978-3-642-42054-2_50

[24] A. Mora-Cortes, N.V. Manyakov, N. Chumerin and M.M. Van Hulle, "Language Model Applications to Spelling with Brain-Computer Interfaces," Sensors 2014, 14, 5967-5993. DOI 10.3390/s140405967.

[25] J. R. Wolpaw, N. Birbaumer, W. J. Heetderks, D. J. McFaralnd, P. H. Peckham, G. Schalk, E. Donchin, L. A. Quatrano, C. J. Robinson, and T. M. Vaughan, "Brain-computer interface technology: A review of the first international meeting," IEEE Trans. On Rehabilitation Engineering, 2000, 8, 164-173. DOI: 10.1109/tre.2000.847807

[26] B. Rivet, H. Cecotti, M. Perrin, E. Maby, and J. Mattout, "Adaptive training session for a P300 speller brain-computer interface", Journal of Physiology-Paris, 2011, 105(1-3), 123-129. DOI: 10.1016/j.jphysparis.2011.07.013

[27] D. Zhang, H. Song, R. Xu, W. Zhou, Z. Ling, and B. Hong, "Toward a minimally invasive brain-computer interface using a single subdural channel: A visual speller study," NeuroImage 2013, 71:30-41.

[28] Y. Liu, Z. Zhou, and D. Hu,"Comparison of stimulus types in visual P300 speller of brain-computer interfaces," IEEE ICCI 2010:273-279.

[29] Y. Shahriari, and A. Erfanian, "Improving the performance of P300based brain-computer interface through subspace-based filtering," Neurocomputing, 2013, 121, 434-441. DOI: 10.1016/j.neucom.2010.01.018

[30] G. Pires, U. Nunes, and M. Castelo-Branco, "Comparison of a rowcolumn speller vs. a novel lateral single-character speller: Assessment of BCI for severe motor disabled patients," Clinical Neurophysiology, 2012, 123(6), 1168-1181. DOI: 10.1016/j.clinph.2011.10.04

[31] L, Acqualagna, B, Blankertz, "Gaze-Independent BCI-Spelling Using Rapid Visual Serial Presentation (RSVP)," Clinical Neurophysiology, 124(5):901-908, 2013. DOI:10.1016/j.clinph.2012.12.050

[32] A. Vilic, T.W. Kjaer, C.E. Thomsen, S. Puthusserypady, and H.B. Sorensen, "DTU BCI Speller: An SSVEP-based Spelling System with Dictionary Support," 35th Annual Int. Conf. of the IEEE EMBS, Osaka, Japan, 3 - 7 July, 2013. DOI: 10.1109/EMBC.2013.6609975.

[33] I. Käthner, C.A. Ruf, E. Pasqualotto, C. Braun, N. Birbaumer, and S. Halder, "A portable auditory P300 brain-computer interface with directional cues," Clinical Neurophysiology, 2013, 124(2), 327-338. DOI: 10.1016/j.clinph.2012.08.006

[34] P.T. Wang, C.E. King, A.H. Do, and Z. Nenadic, "Pushing the Communication Speed Limit of a Noninvasive BCI Speller", CoRR abs/1212.0469 (2012)

[35] S.G. Hart, L.E. Staveland, "Development of NASA-TLX (Task Load Index): Results of empirical and theoretical research," in P.A. Hancock \& N. Meshkati (Eds.), Human mental workload (pp. 139 183). Amsterdam: North-Holland, 1988. 